\title{
Supplementary Information for Monitoring Neuron Activities and Interactions with Laser Emissions
}

\author{
Yu-Cheng Chen ${ }^{1,2}$, Xuzhou Li ${ }^{2,3}$, Hongbo Zhu², Wei-Hung Weng ${ }^{4}$, Xiaotian Tan ${ }^{2}$, Qiushu Chen², \\ Yunlu Sun ${ }^{2}$, Xueding Wang ${ }^{2}$, and Xudong Fan $^{2 *}$ \\ ${ }^{1}$ School of Electrical and Electronics Engineering, Nanyang Technological University, \\ 50 Nanyang Ave, 639798, Singapore \\ ${ }^{2}$ Department of Biomedical Engineering, University of Michigan, \\ 1101 Beal Ave., Ann Arbor, MI, 48109, USA \\ ${ }^{3}$ Department of Mechanical Engineering, University of Michigan, \\ 2350 Hayward, Ann Arbor, MI, 48109, USA \\ ${ }^{4}$ Department of Computer Science, Massachusetts Institute of Technology, \\ Cambridge, MA, USA \\ *Correspondence: xsfan@umich.edu
}

Number of pages: 4

Number of figures: 3 


\section{Optical system setup for laser recording and FP cavity}

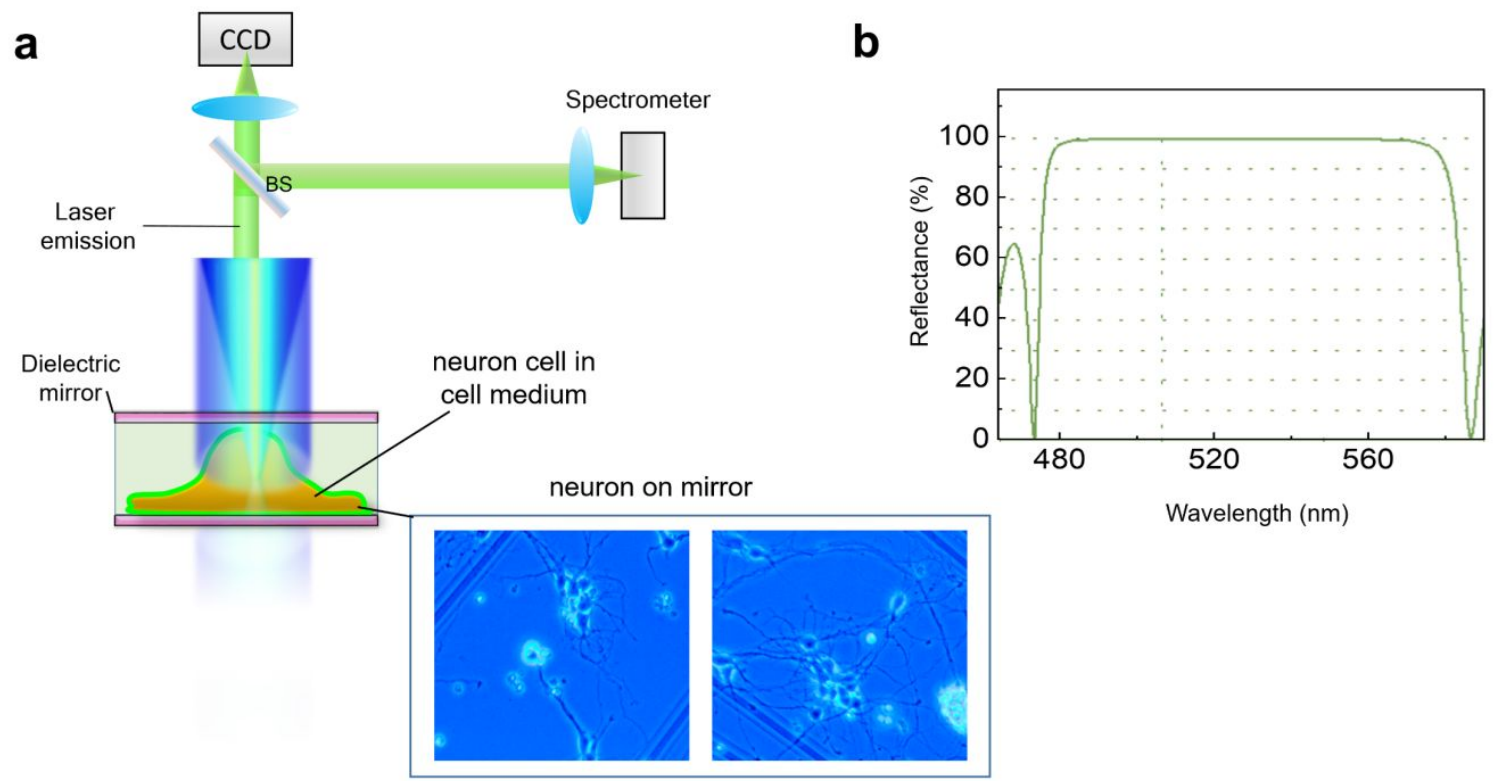

Figure S1. (a) Schematic of the optical experimental setup of the laser-recording system. The focal beam size (spatial sampling area of the spectrum) is $30 \mu \mathrm{m}$ in diameter. The inset figure on the bottom right shows the brightfield images of neurons cultured on mirrors. The neurons were sandwiched between the two mirrors while pumped by a $475 \mathrm{~nm}$ excitation source. (b) The reflectance spectra of the top mirror and the bottom mirror. In this work the top and bottom mirror are the identical design. The reflectivity of the mirrors around the lasing emission wavelength (530$565 \mathrm{~nm}$ ) is measured to be $99.50 \%$. 


\section{Comparison of laser emission and fluorescence}
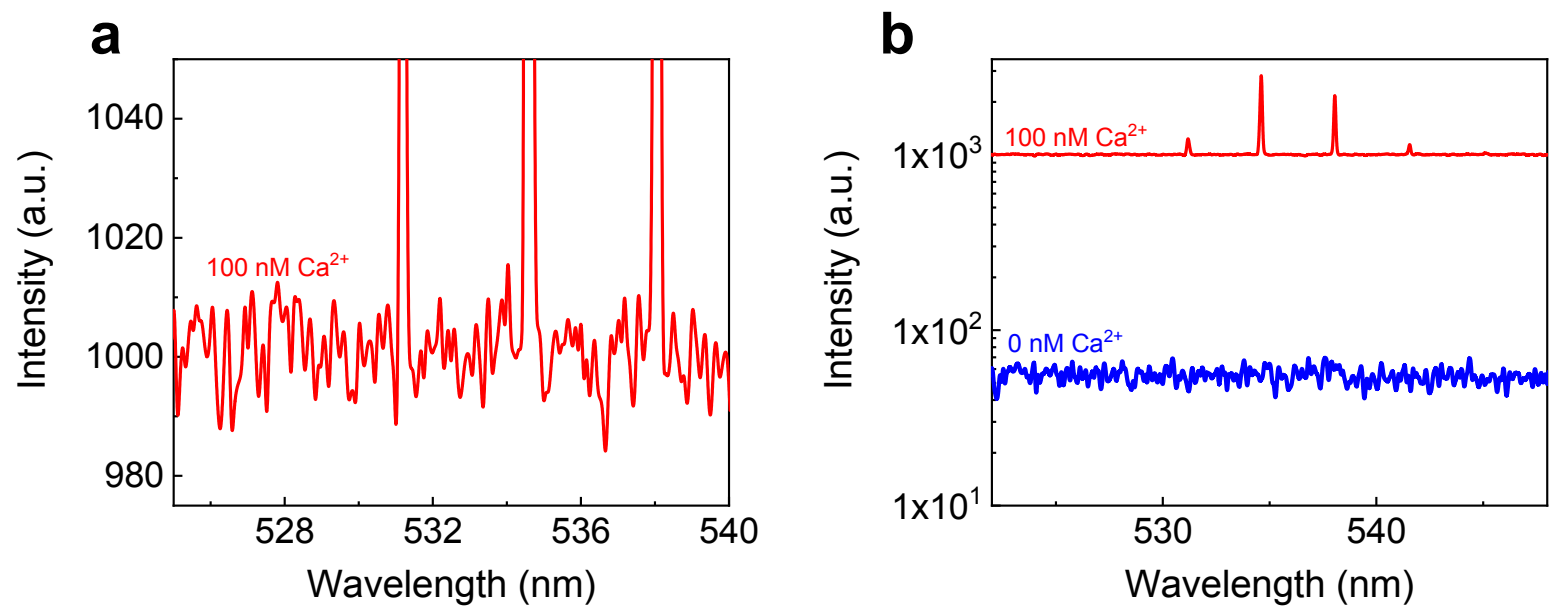

Figure S2. (a) Magnified laser emission spectrum of Fig. 2 in the main text, showing the noise at the baseline. (b) Lasing emission and fluorescence spectra corresponding to Fig. 2 plotted in the logarithmic scale. Note that the baseline is vertically shifted in Fig. 2. 


\section{Comparison of fluorescence recording and laser recording of neuron activities}

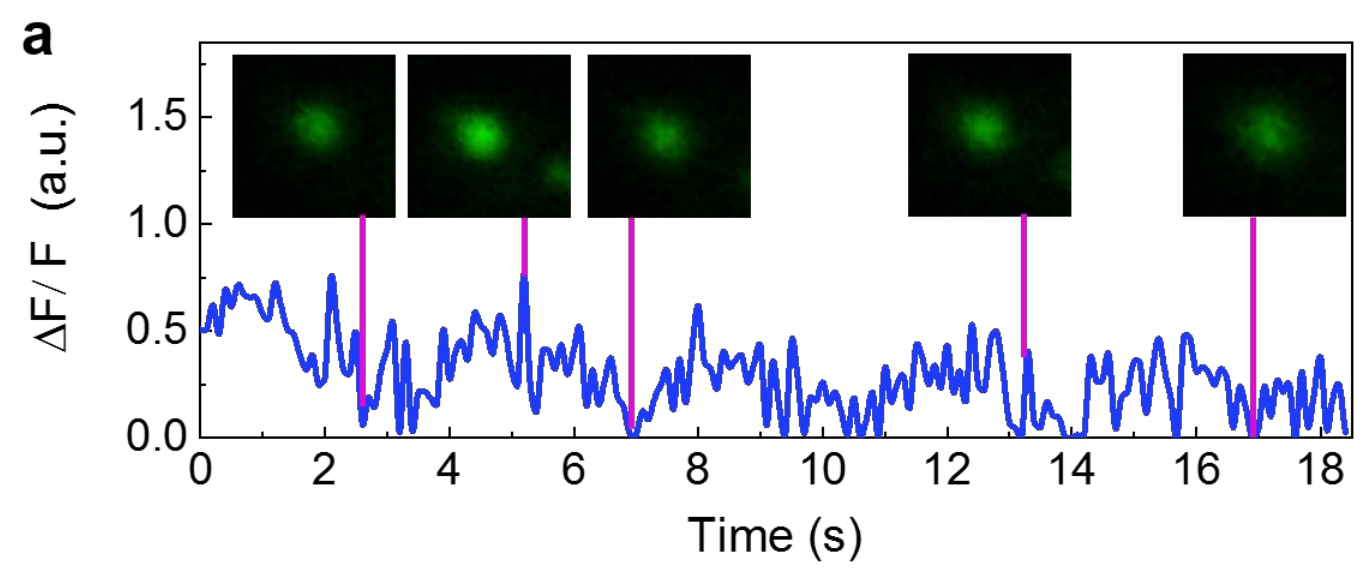

b

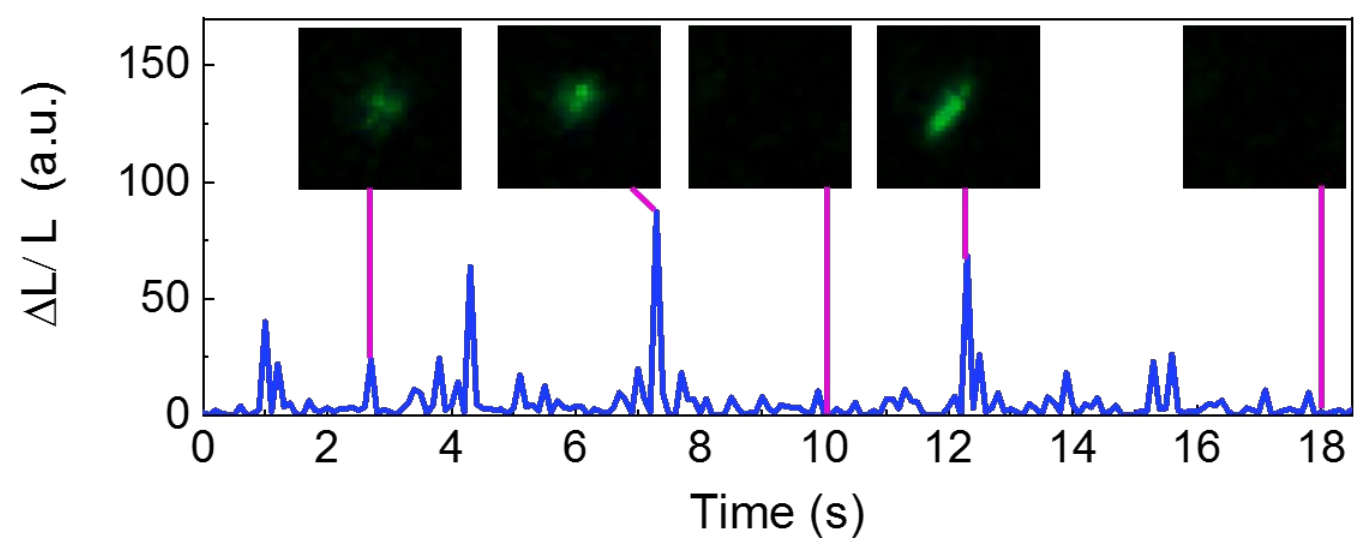

Figure S3. (a) Short-term fluorescence recording of calcium transients caused by spontaneous neuronal activities over 18 seconds from a single neuron. The insets show the representative fluorescence images captured by a CCD camera at $10 \mathrm{fps}$. The SNR is less than 5. (b) Short-term laser recording of calcium transients caused by spontaneous neuronal activities over 18 seconds from a single neuron. The insets show the representative laser emission images captured by the same CCD camera at $10 \mathrm{fps}$. (a) and (b) were not measured simultaneously. Note that the SNR for laser emission images is limited by the dynamic range of the CCD camera, which is different from the traditional images captured by scanning confocal microscopy using a photo-multiplier tube (PMT). Excitation wavelength, $475 \mathrm{~nm}$. Pump energy density, $60 \mu \mathrm{J} / \mathrm{mm}^{2}$. 